\title{
Collision Dynamics of a Water Droplet Impinging on a Rigid Surface above the Leidenfrost Temperature
}

\author{
Natsuo HATTA, Hitoshi FUJIMOTO, Hirohiko TAKUDA, Kenji KINOSHITA ${ }^{1)}$ and Osamu TAKAHASHI
}

Faculty of Engineering, Kyoto University, Yoshida-honmachi, Sakyo-ku, Kyoto, Kyoto-fu, 606 Japan.

1) Graduate School, Kyoto University, Yoshida-honmachi, Sakyo-ku, Kyoto, Kyoto-fu, 606 Japan.

(Received on July 21; accepted in final form on October 21, 1994)

\begin{abstract}
The collision dynamics of a water droplet impinging on a rigid surface above the Leidenfrost temperature are described from an experimental point of view. Emphasis is placed upon the collision of relatively small droplets in the range from 300 to $600 \mu \mathrm{m}$ with the surface. The surface temperature is fixed at $500^{\circ} \mathrm{C}$. The first purpose of the present study is to examine whether or not the prior empirical rules obtained by using droplets of 2 to $3 \mathrm{~mm}$ give an applicability to the case where the droplet size is much smaller. The second is to clarify the relation between the Weber number and the coefficient of restitution, when the droplet rebounds from the hot surface without disintegrating into a number of particles. Also, the maximum value of the diameter of droplet which can spread on the surface and the resident time are examined and discussed by comparing the result obtained here with the prior empirical formulae proposed by a few researchers.
\end{abstract}

KEY WORDS: collision dynamics; water droplet; hot surface; Weber number; droplet deformation; coefficient of restitution.

\section{Introduction}

To predict spray/mist cooling heat transfer, it is indispensable to systematically understand the collision dynamics of liquid droplets on a heated rigid surface beyond the Leidenfrost temperature. Again, because there are a number of parameters affecting heat transfer mechanism between the droplets and the surface and the interaction among of these parameters are very complicated, it is even now a difficult task to evaluate the cooling capacity by a predictable expression.

The collision dynamics of a water droplet on a hot solid surface beyond the Leidenfrost temperature are discussed from an experimental point of view. Now, let it be assumed that a water droplet falls vertically through air and terminates its flight by colliding with a horizontally fixed hot plate surface. After the droplet impinges on the surface it can splash, spread and/or rebound. It is well-known that the phenomenon to be expected may be governed by both the impact energy of the droplet and the temperature of the surface. When the droplet has the low Weber number corresponding to a low impact energy, the droplet may stick and spread and eventually reside as a lens-shaped mass on the surface or rebound as a bowling pin-shaped mass from the surface. While for high Weber numbers the droplet may shatter during the deformation process. According to a few investigations ${ }^{1,2)}$ performed so far it was confirmed that both the maximum spreading diameter on the surface and the boundary whether the droplet rebounds or shatters depend on the Weber number. Also, it was reported $^{3)}$ that the resident time of the droplet on the hot plate surface agrees approximately with the firstorder vibration period of a freely oscillating droplet. These experimental results were derived by using relatively large droplets of $2-3 \mathrm{~mm}$ in diameter. Actually, the droplet diameters in spray cooling are regarded as being in the range between 100 and $500 \mu \mathrm{m}$. It can be doubtful whether the experimental results obtained by using such comparatively large droplets are applicable directly to the case where the droplet size is small in the order of $100 \mu \mathrm{m}$.

Next, we have undertaken the theoretical analysis of mist/spray cooling process to assess the cooling intensity. To do this, we numerically analyzed flow structures of the two-phase air-water droplet mixture jet impinging on a flat plate normal to flow. ${ }^{4,5)}$ However, the numerical calculations were made on the assumption that the droplet reflection from the surface is perfectly elastic. The major reason for introducing the assumption was because the coefficient of restitution remains unclarified between the water droplets and the hot surface. Indeed, we can find some investigations on the collision of a very small droplet with a hot solid surface. Pederson, ${ }^{6)}$ Shoji et $a l .{ }^{7)}$ and Xiong and Yuen ${ }^{8)}$ treated the collision phenomena of fairly small droplets of the order of $100 \mu \mathrm{m}$ on the hot surface. However, they placed emphasis not upon the collision dynamics, but upon the heat transfer between the droplet and the surface. Jeffrey and Patrick ${ }^{9)}$ examined the deformation process of the droplet with the Weber number as the parameter. That is, they inspected the Weber number before and after the colli- 
sion, when the droplet rebounds, and measured the diameter and velocity distributions of individual particles, when the droplet shatters during the deformation process. But the criterion of the rebounding and the disintegrating was not made clear because the experimental condition was restricted to a narrow range.

In the present paper, the collision behaviour of water droplets from 300 to $600 \mu \mathrm{m}$ in diameter on the surface heated at $500^{\circ} \mathrm{C}$ and the rebounding/disintegrating phenomena were investigated experimentally. The first purpose of the present study is to examine whether or not the past empirical rules obtained by using the large droplets of 2 to $3 \mathrm{~mm}$ in diameter give an applicability to the case where the droplet size is much smaller. The second is to clarify the relation between the Weber number and the coefficient of restitution when the droplet rebounds from the heated rigid surface. In this sense, the deformation process of the droplet was measured only on the condition that it can not disintegrated into many particles.

The period required for the droplet of $400 \mu \mathrm{m}$ in diameter to rebound from the surface after the collision is only $1.0 \mathrm{~ms}$ or shorter. Therefore, it is difficult to follow the time-variation of the droplet deformation process, even though the high speed camera capable of taking $10000 \mathrm{frames} / \mathrm{s}$ is adopted. In addition, the droplet behaviour is so susceptible to influences from a very small environmental change that it is uncontrollable by the adjustment of the experimental apparatus. Accordingly, the deformation process of the droplet impinging on the hot surface was measured by a number of video images on the basis of the statistical procedure. The droplet images were exposed twice per frame by two flashes in a certain time interval to catch the variation of the droplet deformation process with time.

\section{Experimental Apparatus}

Figure 1 shows a schematic diagram of the experimental appratus by which the deformation process of a droplet impinging on a heated rigid surface beyond the Leidenfrost temperature can be measured. The water stored in a gastight tank is introduced to a needle and the droplet is formed through the needle. The water is pressurized statically by a high pressure air to ensure repeatability of droplet formation and release at the needle tip. The needle is flat tipped and set vertically. A uniformly spaced stream of droplets is generated with an almost equal diameter and velocity at the needle tip. Also, the adjustment of the spacing of droplets can be made in accord with the vibration frequency of a needle oscillator. A disk of $200 \mathrm{~mm}$ in diameter rotates horizontally between the needle exit and the heated surface. Only one droplet per revolution can pass through the slit hole of the rotating disk and reach the horizontal heated surface. The droplet size as well as the impinging velocity is easily changeable by the water pressure, the vibration frequency of the needle oscillator and the inner diameter of the needle. But, it is impossible to change only the droplet diameter on the condition of keeping the impinging velocity constant and the inverse is also impossible. By this experimental apparatus, droplets of
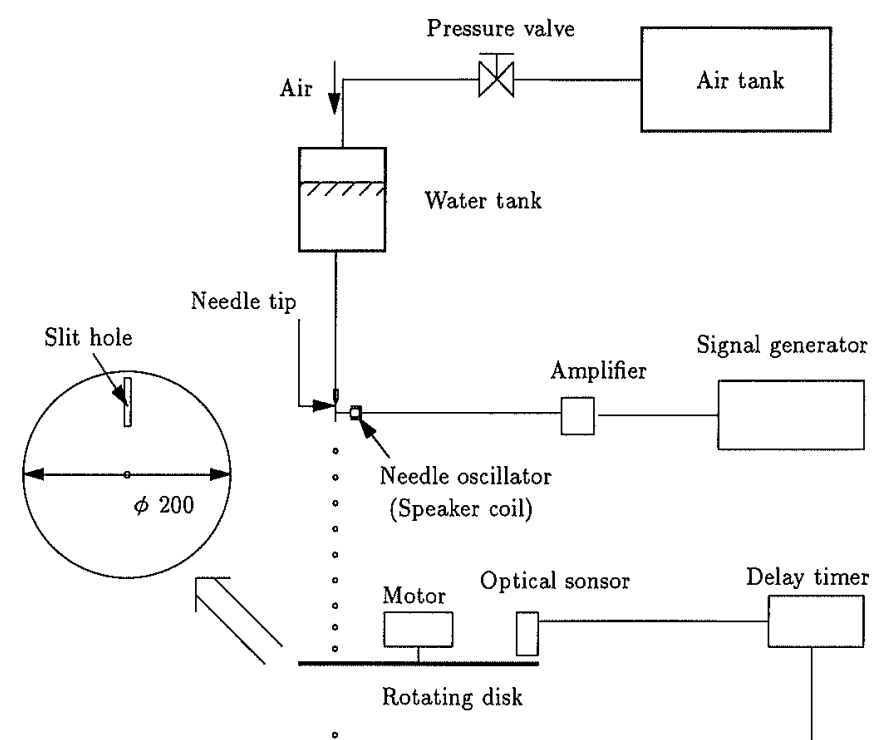

Video camera
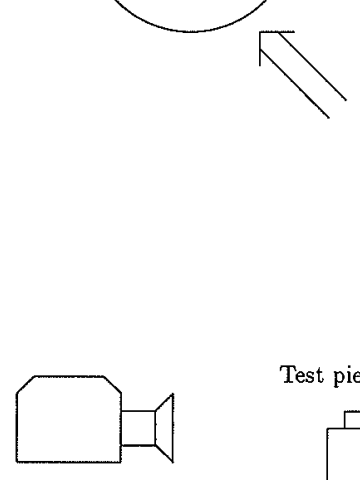

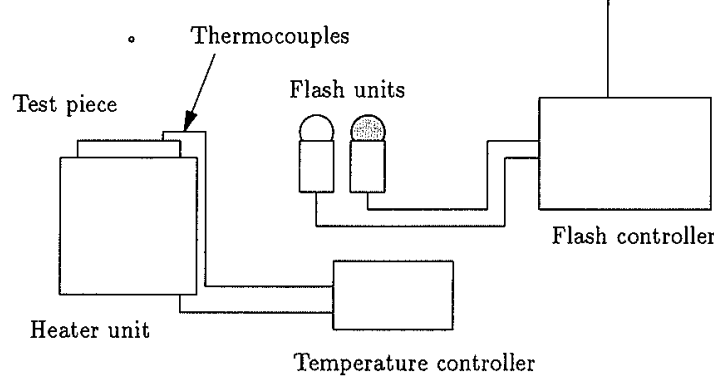

Fig. 1.

Schematic view of experimental apparatus. 


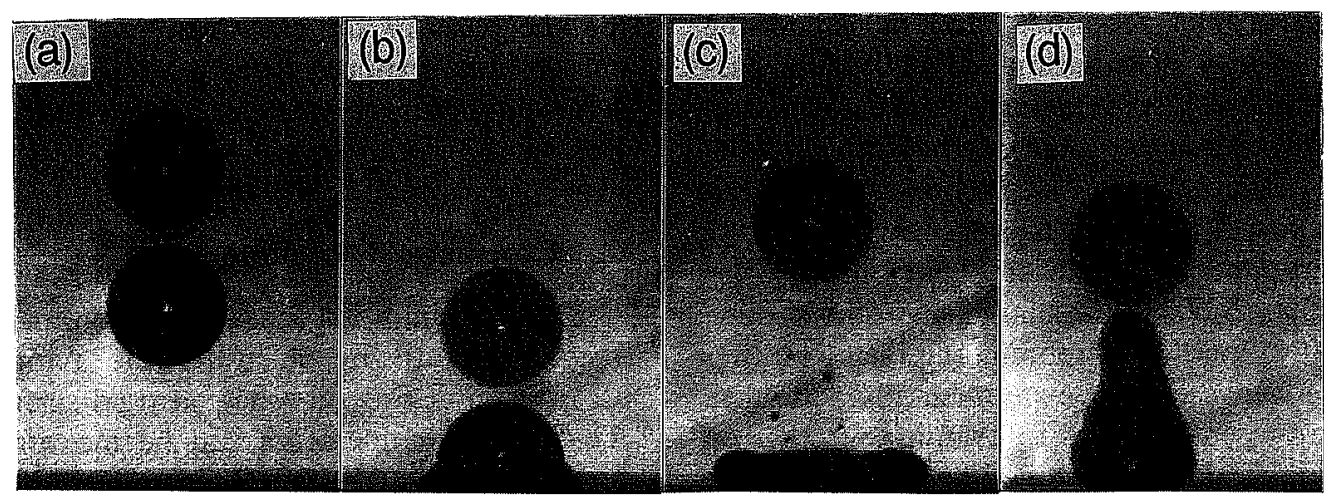

\section{$0.5 \mathrm{~mm}$}

Fig. 2. Droplet deformation process impinging on a plate using two flash units. Note that time interval of two flashes is $200,200,600$ and $800 \mu$ s, for (a), (b), (c) and (d), respectively.

300 to $700 \mu \mathrm{m}$ can be generated in the impinging velocity range between 1.2 and $6.0 \mathrm{~m} / \mathrm{s}$.

The droplet falls vertically onto a $6 \mathrm{~mm}$ thick by $28 \mathrm{~mm}$ diameter Inconel alloy 625 test surface. The test surface temperature is measured by the chromel-alumel thermocouples and adjusted by the thermocontroller unit. Throughout the present paper, the surface temperature is fixed at $500^{\circ} \mathrm{C}$. But, it should be noted that the temperature change during the experiments is confirmed to be within plus or minus $20^{\circ} \mathrm{C}$.

The deformation process of the droplet is recorded by using a video camera which is equipped with a macro lens. The droplet images are exposed by the back light method using two micro flash units, which are scheduled to operate at a constant time interval. The first flash is adjusted to catch the droplet just before the collision with the surface. The flash timing is adjusted by using both an optical sensor perceiving the slit position on the disk and a delay timer. In the present experiments, the droplet is photographed in the range of the time interval from 100 to $1600 \mu \mathrm{s}$. The time interval of the two flash units can be set by a flash controller. The lightening period of the flash units is kept below $2 \mu$ s. The droplet image can be exposed only during flashing. The time variation of the droplet height and width during the deformation is recorded by many double exposure video images on the basis of the statistical procedure. The droplet diameter before reaching the heated surface can be directly measured from the video image. Also, the impinging velocity can easily be estimated from both the moving distance of the droplet and the time interval of the two flashes, a shown in Fig. 2(a). When the impinging velocity is measured, the both images of the same droplet photographed by the first and second flashes are required to be before the collision with the surface, and the time interval of two flashes is adjusted below $200 \mu \mathrm{s}$. The time order of two droplet images can be distinguished by two different color flashes. Figures 2(a)-2(d) is output by the monochrome video printer taken from the color images.

The resident time of the droplet during the deformation on the hot surface after the contact with it can be

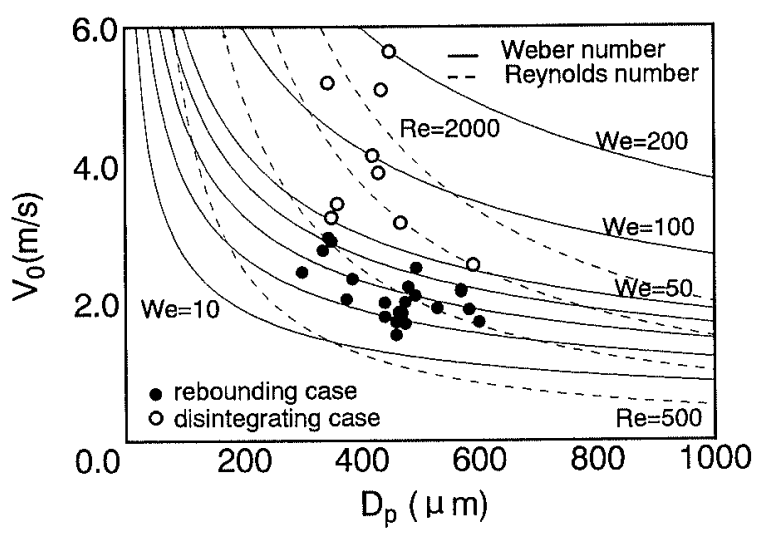

Fig. 3. Rebounding and disintegrating cases with $W e$ and $R e$ as parameters on $V_{0}$ and $D_{p}$ relation.

evaluated from the distance between the droplet and the surface in the first exposed image, the impinging velocity and the time interval of two flashes. Thereby, the height and the width of the droplet on the surface can be measured directly from the second exposed image. The droplet deformation process is pursued by a lot of droplet images, for example, as shown in Figs. 2(a) to 2(d). Here it should be noted that the flashing time intervals of Figs. 2(a) to 2(d) are different from each other, the time interval is 200, 200, 600, $800 \mu$ s for Figs. 2(a), 2(b), 2(c) and 2(d), respectively. Again, the measurement accuracy determined by the resolution of the video image is within plus or minus $0.006 \mathrm{~mm}$ in the present measurement unit.

\section{Results and Discussion}

First, the deformation and rebounding phenomena of the droplet after making contact with the hot surface beyond the Leidenfrost temperature were inspected under the condition that the droplet size ranges from $D_{p}=330$ to $600 \mu \mathrm{m}$ in diameter and the impact velocity is changed in the range from $V_{0}=1.7$ to $5.7 \mathrm{~m} / \mathrm{s}$. The droplet and surface temperatures were kept at 20 and $500^{\circ} \mathrm{C}$, respectively. Figure 3 shows the rebounding and disintegrating cases with the Weber number $W e$ and Reynolds numbers 
$R e$ as parameters on the $V_{0}$ vs. $D_{p}$ diagram, where the solid circles correspond to the rebounding case and the open ones denote the disintegrating case. It is noted that $W e$ as well as $R e$ is represented by the physical properties at $20^{\circ} \mathrm{C}$. There could be observed the droplets which break up into two pieces in air after rebounding and these were, of course, regarded as belonging to the rebounding group. The critical Weber number, which shows the boundary between rebounding and disintegrating, was proposed by some researchers in prior experimental investigations. Ueda et al. ${ }^{2)}$ gave 70 to the critical Weber number on the basis of the experimental results obtained on condition that the droplets of 2 to $3 \mathrm{~mm}$ in diameter fell on the flat stainless steel and copper surfaces heated at $300^{\circ} \mathrm{C}$. Again, Shoji et al..$^{7)}$ made an experimental study on condition that the droplets of 300 to $3530 \mu \mathrm{m}$ impinge on the flat nickel surface heated in the temperature range from 300 to $800^{\circ} \mathrm{C}$ and thereby proposed that the critical Weber number exists in the range between 80 and 90 . According to the present experimental results, the critical Weber number was nearly 50 and relatively small in comparison with the above mentioned two investigations. We consider that the rebounding condition is influenced not only by the Weber number, but also by other parameters such as the surface temperature, the surface roughness and the surface material, etc. As pointed out from an experimental point of view, the Leidenfrost temperature drastically changes with the surface material and roughness ${ }^{10)}$ and the boiling phenomenon of the droplet is also changeable depending upon such parameters.

Thus, on the basis of the present result, we tried to investigate the deformation behaviour of the droplet on the Inconel alloy 625 surface heated at $500^{\circ} \mathrm{C}$ in the range of $W e$ smaller than 50 . Figure 4 shows the typical time

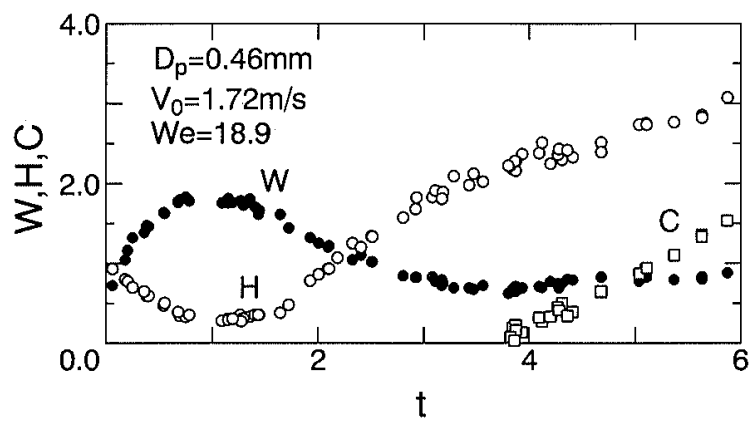

Fig. 4. Time history of deformation process of droplet on condition that $D_{p}=0.46 \mathrm{~mm}$ and $V_{0}=1.72 \mathrm{~m} / \mathrm{s}$.

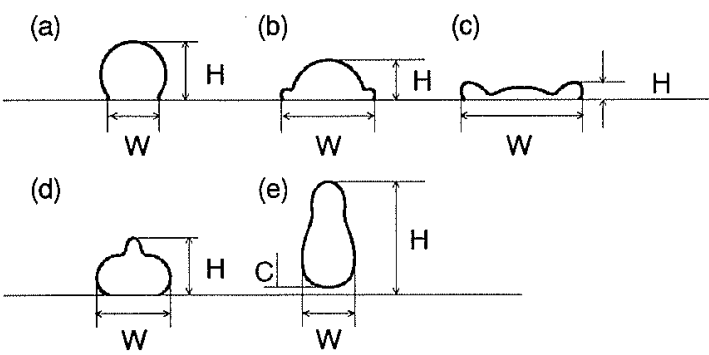

Fig. 5. Definition of droplet height $H$, width $W$ and distance $C$ from bottom of droplet to plate surface after rebounding from surface. history of the deformation process of the droplet after the collision with the surface under the experimental condition that $D_{p}=0.46 \mathrm{~mm}$ and $V_{0}=1.72 \mathrm{~m} / \mathrm{s}(W e=$ 18.9 and $R e=791) . H, W$ and $C$ denote the droplet height, the width and the distance between the droplet bottom and the surface after rebounding from the surface, respectively (see Fig. 5). Here, it should be noted that $H$, $W$ and $C$ are non-dimensionalized by the initial spherical droplet diameter $D_{p}$ and $t$ is done by $D_{p} / V_{0}$. By the way, $t=1$ corresponds to $267 \mu \mathrm{s}$. Again, the variations of $W$, $H$ and $C$ with $t$ in Fig. 3 were pursued by many video images of droplets, as has been mentioned already. In an early stage of $t<0.8, H$ decreases at the almost constant rate which is equal to approximately the impact velocity, while $W$ increases with time. After the doplet height $H$ reaches the minimum value around $t=1.0$, its increasing rate is very slowly for a short time. After $t \simeq 1.7$ the increasing rate of $H$ becomes remarkable suddenly. On the other hand, $W$ reaches the maximum value around $t=1.0$ and thereafter continues to decrease. But, after $t \sim 3.5$, there is observed almost no notable change in $W$. Here, what is worthy of attention is that the droplet rebounds from the surface around $t=3.8$ when the droplet length $(=H-C)$ is approximately 2.2 times the initial droplet diameter $D_{p}$. However, the droplet tends to shorten as time elapses. It may be reasonable to consider that the upward velocity of the bottom side of the droplet becomes larger than that of the top side.

It should be noted that in a later stage after the collision with the surface the measured values tend to be scattered. This tendency, becomes remarkable as the Weber number is increased. The major reason is due to the fact that most of droplets can not keep the shape axially symmetric when the droplets are lengthened as a bowling pin-shaped mass on the surface. Figure 6 indicates the time history of the deformation process of the droplet after the collision with the surface on condition that $D_{p}=0.35 \mathrm{~mm}$ and $V_{0}=2.9 \mathrm{~m} / \mathrm{s}(W e=41$ and $R e=1015)$. The dispersion of the measured values of $W, H$ and $C$ after rebounding from the surface becomes obviously remarkable compared with the case shown in Fig. 4.

The maximum spreading diameter of droplets impinging on a high temperature surface was investigated experimentally by a few researchers. The empirical formulae were proposed in the form of the function of only the Weber number. Akao et al. ${ }^{1)}$ proposed the following form of

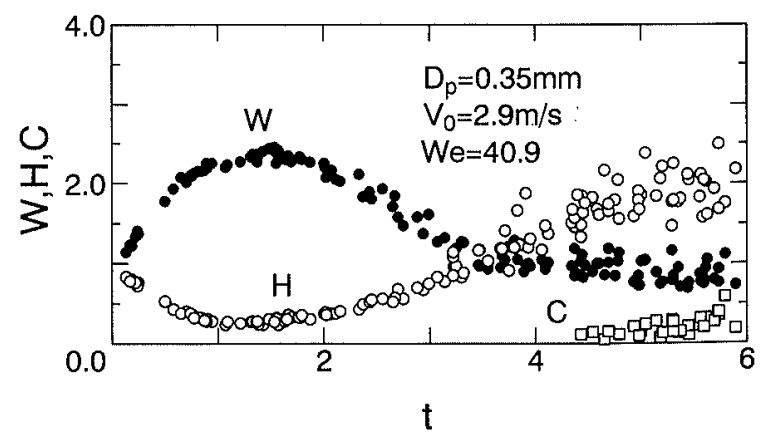

Fig. 6. Time history of deformation process of droplet on condition that $D_{p}=0.35 \mathrm{~mm}$ and $V_{0}=2.9 \mathrm{~m} / \mathrm{s}$. 


$$
D_{\max } / D_{p}=0.613 W e^{0.39}
$$

and Ueda $e t a l{ }^{2)}$ gave the following relation as

$$
D_{\max } / D_{p}=0.87\left(\frac{W e}{6}+2\right)^{0.5}
$$

The above two expressions were obtained using the relatively large droplets of the order of a few millimeters in diameter.

Figure 7(a) indicates the relation between $D_{\max } / D_{p}$ and $W e$, where $D_{\max }$ denotes the maximum spreading diameter after the collision with the surface. The solid circles show the present experimental results obtained by using droplets of 330 to $600 \mu \mathrm{m}$ in diameter. Also, the open circles denote the results given by Ueda et al., which were obtained by using droplets of 0.94 to $2.5 \mathrm{~mm}$ in diameter at the saturation temperature of water. Therefore, the Weber number was estimated by the physical properties at $20^{\circ} \mathrm{C}$ for the present case and at $100^{\circ} \mathrm{C}$ for the case of Ueda et al.

The above mentioned two experimental formulae are found to give good agreement with each other in the range of the Weber number larger than 25 . The present experimental data is also seen to be fairly close to these formulae, but the values are slightly small in comparison with the two formulae. Then, because it was considered that the maximum spreading diameter depends not only upon the Weber number, but also upon the Reynolds number, the effect of the Reynolds number on $D_{\max }$ was inspected by plotting the value of $D_{\max }$ against the Reynolds number. As a result, almost no significant effect could be found. Then the formula capable of giving best-fit to the present experimental data was built up in
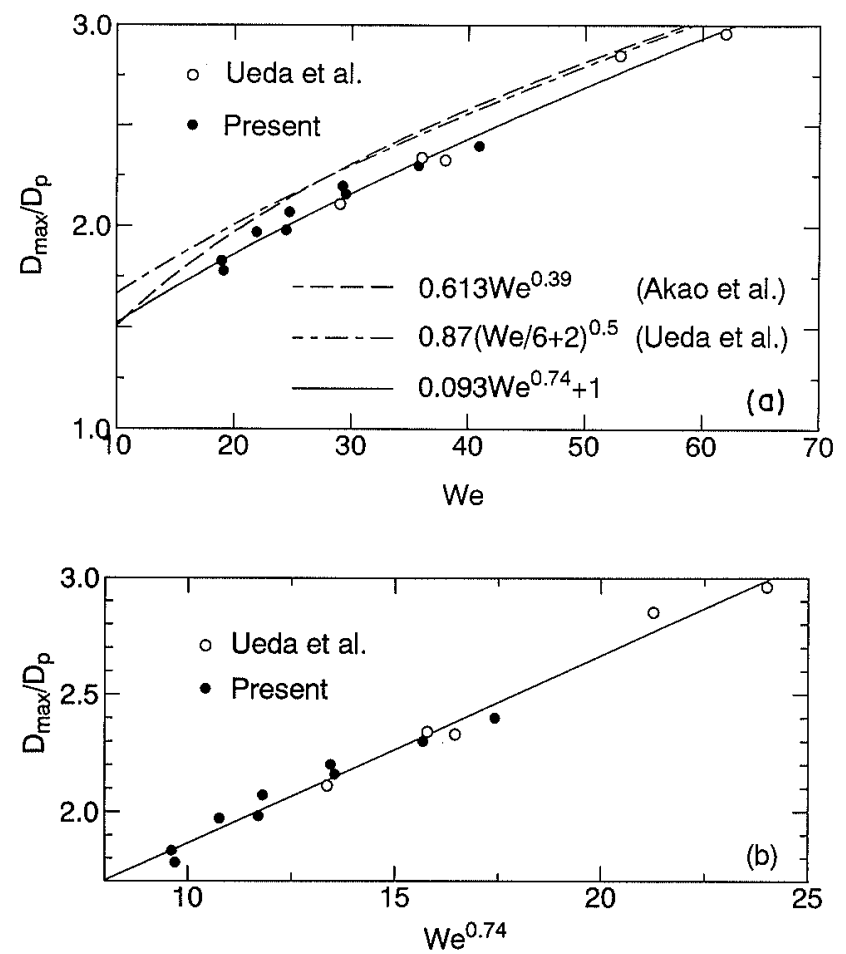

Fig. 7. Relation between maximum spreading diameter after collision with surface and the Weber number (a) and comparison of formula built up in present study with measured values (b). the form of

$$
D_{\max } / D_{p}=0.093 W e^{0.74}+1
$$

The numerical value obtained by the above expression could be found to give good agreement not only with the present data, but also with the results by Ueda $e t$ al. Such a relation may evidently be understood in Fig. 7(a) as well as Fig. 7(b).

Wachters et al. ${ }^{3)}$ and Ueda et al. ${ }^{2)}$ reported that the resident time of droplets on the hot surface is approximately equal to the first-order vibration period $t$ of a freely oscillating droplet given in the form of

$$
t=\frac{\pi}{4} \sqrt{\frac{\rho D_{p}^{3}}{\sigma}}
$$

in which $\rho$ and $\sigma$ denote the density and the surface tension, respectively. Shoji et al. ${ }^{7)}$ confirmed experimentally that Eq. (4) holds true for relatively small droplets of the order of $100 \mu \mathrm{m}$. Figure 8 gives the variation of the resident time with the droplet size. It was found that from this figure that that values obtained by Eq. (4) are in good agreement with the measured resident time of droplets in the size range of 0.33 to $0.48 \mathrm{~mm}$. Concretely speaking, the resident time is estimated to be only $500 \mu \mathrm{s}$ for $D_{p}=0.3 \mathrm{~mm}$ and $1 \mathrm{~ms}$ for $D_{p}=0.5 \mathrm{~mm}$. It may be understood that as soon as the droplet impinges on the hot surface, it can be instantaneously in direct contact with the surface and after a very short wetting time the droplet is isolated from the surface through a vapour

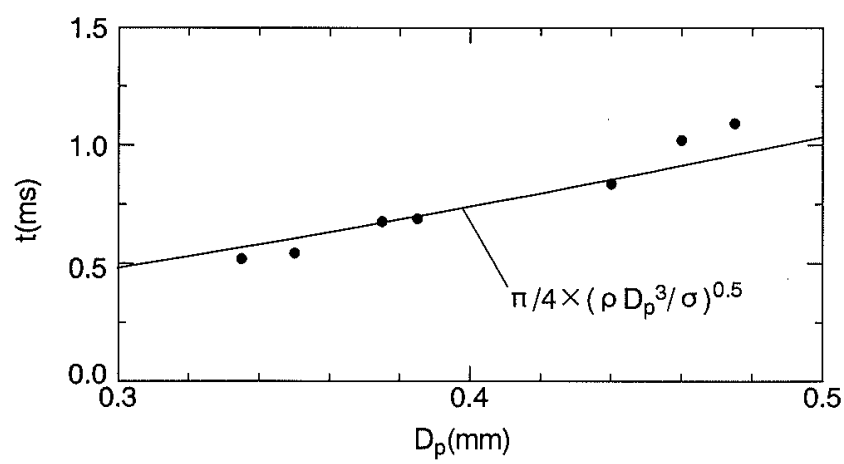

Fig. 8. Variation of resident time with droplet size.

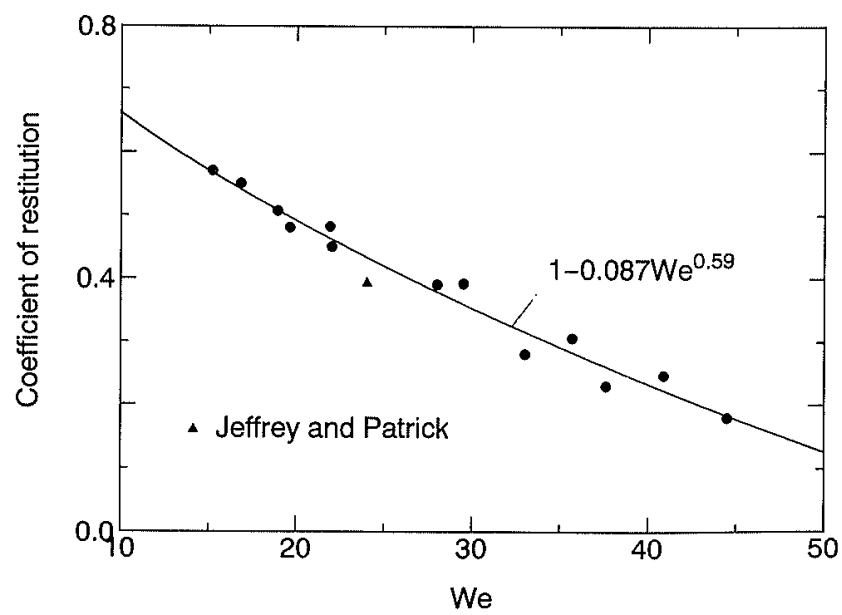

Fig. 9. Coefficient of restitution of droplet against Weber number in size range of $D_{p}=0.33$ to $0.48 \mathrm{~mm}$. 
film. Therefore, it is very difficult to quantitatively assess the effect of the wetting on the cooling intensity from a thermal point of view.

Figure 9 shows the coefficient of restitution of droplets against the Weber number in the size range of $D_{p}=0.33$ to $0.48 \mathrm{~mm}$. As droplets are not spherical in shape after impact on the surface, but lengthened as a bowling pin-shaped mass on rebounding from the surface, the coefficient of restitution $e$ is defined in the form,

$$
e=\left|\frac{0.5\left(V_{\text {top }}+V_{\text {bottom }}\right)}{V_{0}}\right|
$$

in which $V_{\text {top }}$ and $V_{\text {botom }}$ are the rebounding velocity at the top and bottom parts of the droplet, respectively. The velocities were determined directly by following the variation of $H$ and $C$ with time at the moment when the droplet leaves the surface (see Fig. 4). It is understood from this figure that the coefficient is more than 0.5 in the range of $W e<18$ and becomes small with the Weber number. There has been almost no measured records of the coefficients of restitution, so far as we know. Only Jeffrey and Patrick ${ }^{9)}$ measured it on condition that the water droplet of $D_{p}=302 \mu \mathrm{m}$ impinging on a steel surface heated at $400^{\circ} \mathrm{C}$. They reported that the average rebounding velocity was $0.94 \mathrm{~m} / \mathrm{s}$ for $V_{0}=2.4 \mathrm{~m} / \mathrm{s}$. The measured result $(e=0.392$ at $W e=24)$ agrees well with the present experimental data, as shown in Fig. 9. Finally, we wish to propose the empirical formula giving the relation between the coefficient of restitution and the Weber number as

$$
e=1-0.087 W e^{0.59}
$$

in the present experimental range. It is noted that the solid line drawn in Fig. 9 obeys the above expression.

\section{Conclusion}

The deformation process as well as the rebounding one of water droplets from 300 to $600 \mu \mathrm{m}$ in diameter impinging on the rigid surface heated at $500^{\circ} \mathrm{C}$ were investigated experimentally. The impact velocity was changed in the range from $V_{0}=1.7$ to $5.7 \mathrm{~m} / \mathrm{s}$. The results obtained in the present study are summarized as follows:

(1) The critical Weber number, which corresponds to the boundary whether the droplet breaks up on the surface or not, was nearly 50 in the present experiments and fairly small in comparison with prior experimental results proposed by a few researchers.

(2) The maximum spreading diameter of droplets on the surface was inspected. The present data was found to give fairly good agreement with the results obtained by the prior two experimental formulae. But, the present data were slightly small in comparison with two formulae (see Fig. 7(a)). Accordingly, the formula capable of giving best-fit to the present data was built up as a function of the Weber number. The value obtained by this formula was found to be in good agreement not only with the present data, but also with the results by the other researchers (see Fig. 7(b)).

(3) The variation of the resident time of droplets on the surface with the droplet size was confirmed to be in good agreement with the first-order vibration period of freely oscillating droplet.

(4) The coefficient of restitution was found to be more than 0.5 for $W e<18$ and to decrease with the Weber number. Also, the empirical formula capable of predicting the coefficient of restitution in the range of $W e<50$ was proposed in the form of a function of the Weber number.

\section{Acknowledgments}

The authors would like to note that this study was supported through the Grant in Aid for Scientific Research (04555163) of the Ministry of Education, Science and Culture in Japan and the Kawakami Memorial Foundation.

\section{REFERENCES}

1) F. Akao, K. Araki, S. Mori and A. Moriyama: Trans. Iron Steel Inst. Jpn., 20 (1980), 737.

2) T. Ueda, T. Enomoto and M. Kanetsuki: Bull. Jpn. Sci. Mech. Eng., 22 (1979), 724.

3) L. H. J. Wachters and N. A. Westerling: Chem. Eng. Soc., 21 (1966), 1047.

4) N. Hatta, H. Fujimoto and R. Ishii: ISIJ Int., 31 (1991), No. 4, 342.

5) N. Hatta, H. Fujimoto and H. Takuda: Appl. Sci. Res., 50 (1993), 129.

6) C. O. Pedersen: Int. Heat Mass Transfer, 13 (1970), 369.

7) M. Shoji, T. Wakunaga and K. Kodama: JSME-B, 50-451 (1984), 716.

8) T. Y. Xiong and M. C. Yuen: Int. J. Heat Mass Transfer, 34-7 (1991), 1881

9) D. N. Jeffrey and V. F. Patrick: SAE Technical Paper 930919 , (1993).

10) S. Chandra and C. T. Avedisian: Int. J. Heat Mass Transfer, 35-10 (1992), 2377. 